\title{
Model predictive control of a crude oil distillation column
}

\author{
MORTEN HOVD*, RUNE MICHAELSEN† and TOMAS MONTIN:
}

Keywords: Modelling, model updating, TBP curve, consistent specifications

The project of designing and implementing model based predictive control on the vacuum distillation column at the Nynäshamn Refinery of Nynäs $\mathrm{AB}$ is described in this paper. The paper describes in detail the modeling for the model based control, covers the controller implementation, and documents the benefits gained from the model based controller.

\section{Introduction}

Nynäs $\mathrm{AB}$ is an oil refining company which focuses on the production of bitumen and various industrial oils. The industrial oils are used in a variety of products, such as transformer oil, lubricants or as a basis for printing ink. There are strict quality specifications for all of Nynäs AS's products. In addition to the stringent quality demands, the capacities of Nynäs' refineries are throughout much of the year constrained by the crude oil distillation towers.

With this background, Nynäs AB entered into a project with Fantoft Prosess at the end of 1994, with the aim of implementing advanced model based control of the crude oil distillation tower at the Nynäshamn Refinery. This paper describes the development of the model based controller, with emphasis on the modelling of the process.

\section{Process description}

A simplified schematic drawing of the distillation column is shown in Fig. 1. The feed to the distillation tower is first heated with heat exchange with the hot product streams, and then further heated in a furnace before being fed to the column. The top product and four side products are withdrawn above the feed plate, and only the bottom product, bitumen, is withdrawn below the feed plate. The boilup in the bottom of the distillation column is provided by injection of superheated steam, as is common in crude oil distillation. The vapour in the top of the column is condensed, and in the accumulator the water and hydrocarbons split into two immiscible liquid phases. The water in the accumulator is transferred to waste water treatment. The hydrocarbon phase (the naphtha) provides the reflux to the column, and a product naphtha stream is withdrawn. The side products are termed "fractions" $1-4$, with fraction 1 being the upper side product. Each of the four side products are stripped with stream in before being transferred to storage, this allows for adjustments to their flash points.

*Author to whom all correspondence should be addressed. Fax: ( + 47) 73 594399; Phone: (+47) 73 591426; E-mail: morten.hovd@itk.ntnu.no

†Fax: ( + 47) 67 153112; Phone: (+ 47) 67 153111; E-mail: rmichae@online.no

†Fax: (+ 46) 8520 14706; Phone: $(+46) 852065000$; E-mail: tomas.montin@nynas.se

Reprinted from Computers and Chemical Engineering, Vol. 21, Morten Hovd, Rune Michaelsen and Tomas Montin, Model predictive control of a crude oil distillation column, 1997, with permission from Elscvicr Scicnce. 


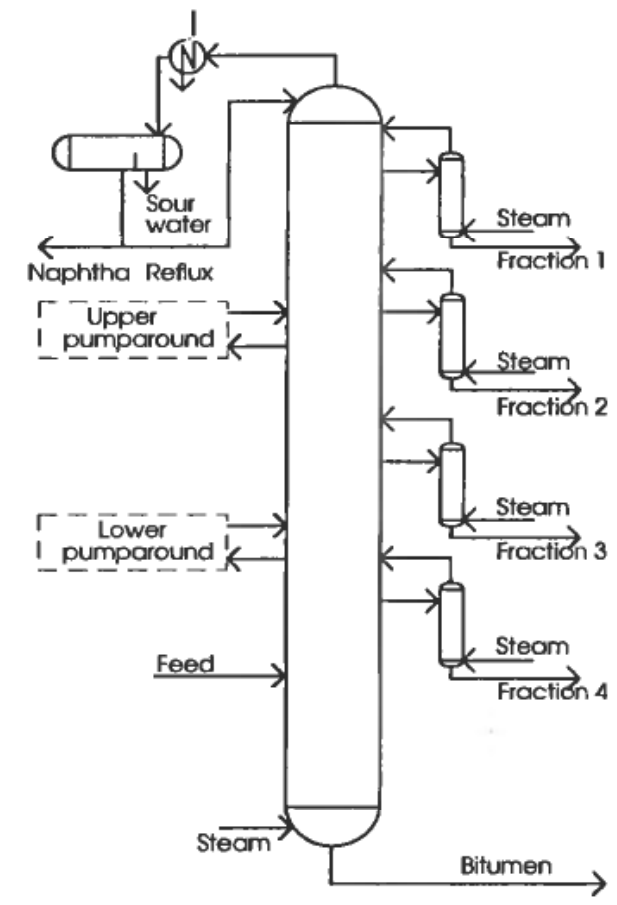

Figure 1. Simplified schematic outline of Nynäshamn distillation column.

The upper and lower pump-arounds, where liquid is withdrawn from the column and heat exchanged before being returned to the column, are not shown in any detail in Fig. 1. These pump-arounds, and the capacity of the condenser for the top product, are important constraints when maximising throughput and optimising energy efficiency, but are not used for control of product quality or yield. The use of these variables will therefore not be discussed in any detail in this paper.

\section{Description of control problem}

The reflux in the top of the column is used to control the temperature in the top of the column. The set-point for the temperature is set to obtain a specified flowrate for the naphtha stream, typically $0.5 \%$ of the feed flowrate, the exact value being determined by flash point specifications for fraction 1 . The feed rate is continuously being maximised, and the flow rate for bitumen must therefore be used to control level in the bottom of the column. This leaves four variables for product quality or yield control, these are the flowrates of fractions $1-4$.

With four manipulated variables, four product qualities or yields can be controlled. The number of candidate controlled variables is however significantly higher:

- The flash points of fractions 2-4 and the bitumen, the flash point of fraction 1 being determined by the naphtha yield.

- The viscosities of fractions 1-4 and the bitumen.

- The yields of fractions 1-4 and the bitumen, the yield of a product being defined as the product flowrate divided by the feed flowrate.

This gives a total of 14 candidate controlled variables, but no more than four of these can be controlled at the same time. One objective for the controller design was that the controller should handle any allowable combination of four candidate controlled 


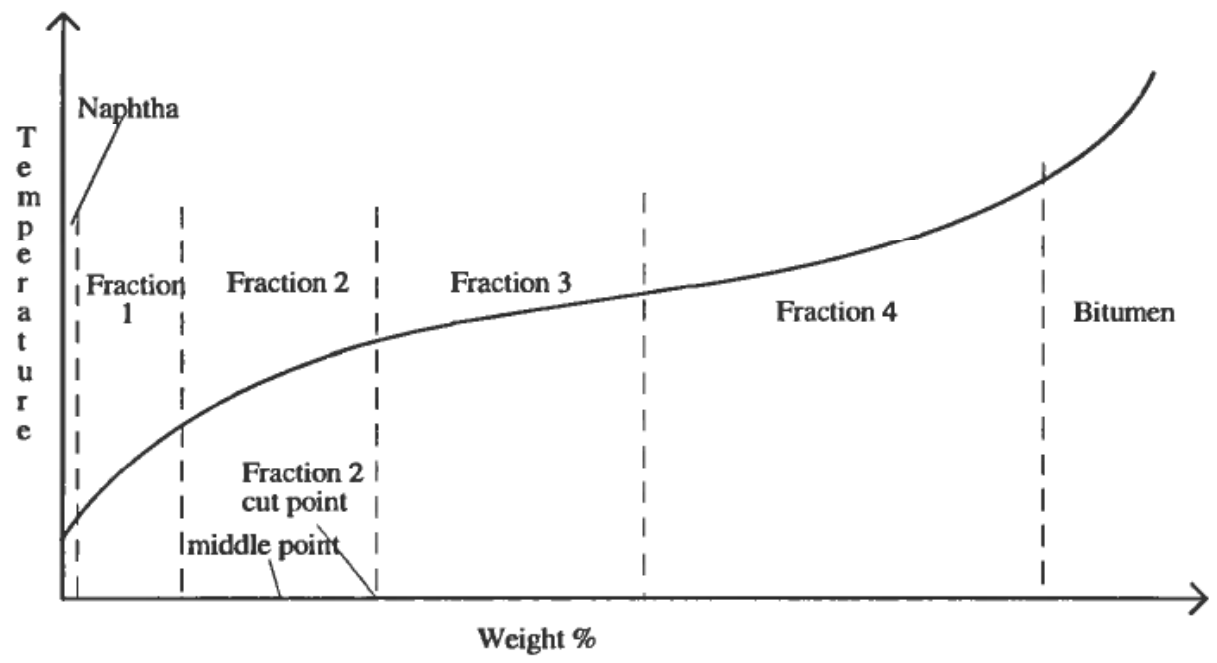

Figure 2. Illustration of True Boiling Point (TBP) curve.

variables from the set of 14 candidate controlled variables. What constitutes an allowable combination of controlled variables is explained in the next section.

Inspection of the online flash point analysers revealed that these were unreliable, and could misfunction for significant periods of time. It was therefore decided not to use the flash points directly as controlled variables, but rather transform a flash point specification to a corresponding product yield specification. This reduces the number of candidate controlled variables to 10 , but it is still necessary to have a stationary model for the product flush points to be able to convert from flash point specifications to yield specifications. The model for the product flash points can be updated whenever required, provided the column is close to stationary and the flash point analysers are functioning.

\section{Consistency of control specifications}

An important laboratory experiment that provides information about the properties of the crude oil is the so-called True Boiling Point (TBP) curve. The TBP curve will be discussed in some detail since it provides important insight about the crude oil distillation process. The TBP curve shows how the boiling point of the remaining crude oil increases as an increasing fraction of the crude oil is removed by distillation. This is shown in a curve with the weight \% distillate on the $\mathbf{x}$-axis and the corresponding boiling point temperature of the remaining crude oil on the $y$-axis, as illustrated in Fig. 2 . The different products from the crude oil distillation column can be drawn into a TBP diagram, the top (naphtha) product occupying the part of the $\mathrm{x}$-axis adjacent to the origin, followed by fractions 1-4 in sequential order, and finally the bottom (bitumen) product. The width of each product section of the $\mathrm{x}$-axis corresponds to the percentage yield of that product. This is illustrated in Fig. 2. The point on the $x$-asix where product $n$ ends and product $n+1$ starts, is called the cut-point of product $n$, and the corresponding temperature the cut-point temperature.

Not only the yield, but also the other candidate controlled variables (viscosity and flash point) are related to the TBP curve. The viscosity of product $n$ is related to its mid-point temperature, i.e, the temperature corresponding to the point halfway between the cut-points of products $n-1$ and $n$. The flash point of product $n$ is related to the cut-point temperature of product $n-1$. 
Since different products cannot overlap on the TBP curve, it is obvious that yield, flash point and viscosity of a product cannot all be controlled variables at the same time, only two of these three variables can be controlled for any given product. More complex combinations of specifications can also be infeasible (i.e., both yield and viscosity of two adjacent products). It should also be noted that the flash point of fraction 1 is determined by the naphtha yield (typically $0.5 \%$ ) and that the "cut-point" of the bitumen is always $100 \%$.

\section{Choice of modelling method}

It was decided at an early stage that it was not feasible to identify models from plant experiments that would cover all the possible operating scenaria for the distillation column, since the experimental work involved would be excessive. Some reasons for this are:

1. The process is non-linear, and the optimal operating point depends strongly on the market prices for the different products, which vary with time. It is possible that a model identified for one operating point would be acceptable at other operating points, but this would be difficult to know à priori.

2. There is occasionally a capacity constraint in Nynäs AB's Gothenburg refinery. In such cases, distillate is transported from Gothenburg to Nynäshamn, to be mixed with the fresh feed to the crude oil distillation column. This has a very strong effect on the operation of the column.

3. There are a number of different operating modes for the column, depending on what product yields and properties are being optimised.

It is also important to realise that a truly rigorous model of crude oil distillation is impossible, since the number of different chemical species is immense, and there does not even exist any analysis method which is able to distinguish between and identify all the chemical species. There exists semi-rigorous steady state simulation programs which uses the results from laboratory experiments to predict product qualities and yields in crude oil distillation. Since such simulation programs cannot give better results than the laboratory data they rely on, it was decided to base the model on the direct use of the laboratory data.

\section{Control model}

First, the TBP curve was obtained experimentally. Then the following relationships were determined experimentally:

1. Flash points as functions of the cut-point temperature of the fraction immediately above the fraction in question.

2. Viscosities as functions of fraction mid-point temperatures.

The relationship between the TBP curve and flash points and viscosities were determined by taking numerous samples from the distillate from the TBP curve experiment. For these samples, flash points and viscosities were measured.

By curve fitting the TBP curve, viscosity and flash point curves, a non-linear steady state model of the distillation column is obtained. From the TBP curve, and the relationship between flash points and cut-point temperatures, as well as the relationship between viscosities and mid-point temperatures, it is evident that the steady state gain matrix is always lower triangular. This is because, if the yield of a given fraction is increased, cut-points and mid-points of the lighter fractions are unaffected. 


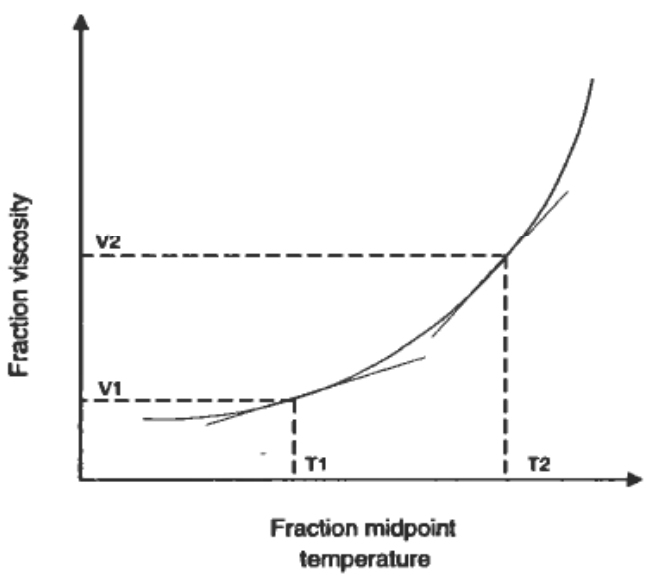

Figure 3. Correction of steady state model based on process measurements.

When an operator enters a new set of specifications for control, these specifications are first checked for consistency, as explained above. If the specifications are consistent, the non-linear model obtained by curve fitting the experimental data is solved to find the corresponding yields of fractions 1-4. Any flash-point specification is then replaced by an equivalent yield specification.

The non-linear model is then perturbed to find a steady state gain matrix. However, since the non-linear model is not perfect, it must be updated with measurements from the plant. Since the TBP curve is per definition a steady state relationship, the model update can only be performed when the column is close to steady state. How this is done will next be explained for the case of a viscosity specification, with the aid of Fig. 3 . Correction of the flash-point model is performed similarly.

The TBP curve gives the relationship between the mid-point of the product and the corresponding temperature. Let this mid-point temperature be termed $T 1$. The product viscosity can then be calculated as a function of the mid-point temperature, by using the relationships extracted from the laboratory experiments, the result of this calculation is the viscosity $V 1$. However, due to inaccuracies in the model, the calculated viscosity will not correspond to the viscosity that is measured at the plant, the viscosity measured at the plant, $V 2$, will instead correspond to a temperature $T 2$. Since viscosity is a non-linear function of mid-point temperature, the gradient at temperature $T 1$ differs from the gradient at temperature $T 2$. A more accurate gain is obtained by perturbing the model around $T 2$ than around $T 1$. This is achieved by adding $T 2-T 1$ to the mid-point temperature found from the TBP curve.

After obtaining the gain matrix, estimated plant dynamics are added. The plant dynamics are chosen to be of the type first order response + time delay. The time constants and time delays will depend largely on liquid distribution and flows within the column, and will vary with operating point. The engineers chose approximate values for time delays and time constants, based on experience from the plant-in pactice rather conservative values are chosen, i.e., slow responses.

The practice of blending distillate from the Gothenburg refinery into the feed of the Nynäshamn distillation column represents a very large feed composition disturbance. The TBP curve used in the model therefore has to be updated accordingly, since the distillate is much lighter than the fresh feed. However, it has not been found necessary 
to modify the viscosity and flash-point curves-but this finding clearly depends on the fact that the Gothenburg refinery uses the same crude oil as in Nynäshamn.

\section{Implementation}

The model based control is implemented using Fantoft Prosess' package D-MPC. This package is very flexible both with respect to model representation, updating from measurements, and taking account of physical constraints in the plant. However, in this application D-MPC used the linear model obtained by linearising the non-linear model, and the calculation of new manipulated variables then becomes very similar to what is conventional. The calculation of manipulated variables is formulated as a QP problem, where the criterion to be optimised involves predicted offsets from set-point in the controlled variables and changes in the manipulated variables. The first change in the manipulated variables is implemented, and the QP problem solved once every time step (which is 10 minutes). Here, a simple bias update is used to update the model with plant measurements.

D-MPC is run on a VAX computer, which communicates with the DCS from Fisher-Rosemount. All parameters needed to define the control problem are stored in an ORACLE database. These parameters are changed by the operators whenever the control specifications change. This is done directly from the DCS terminals, where special dynamic ORACLE forms can be shown.

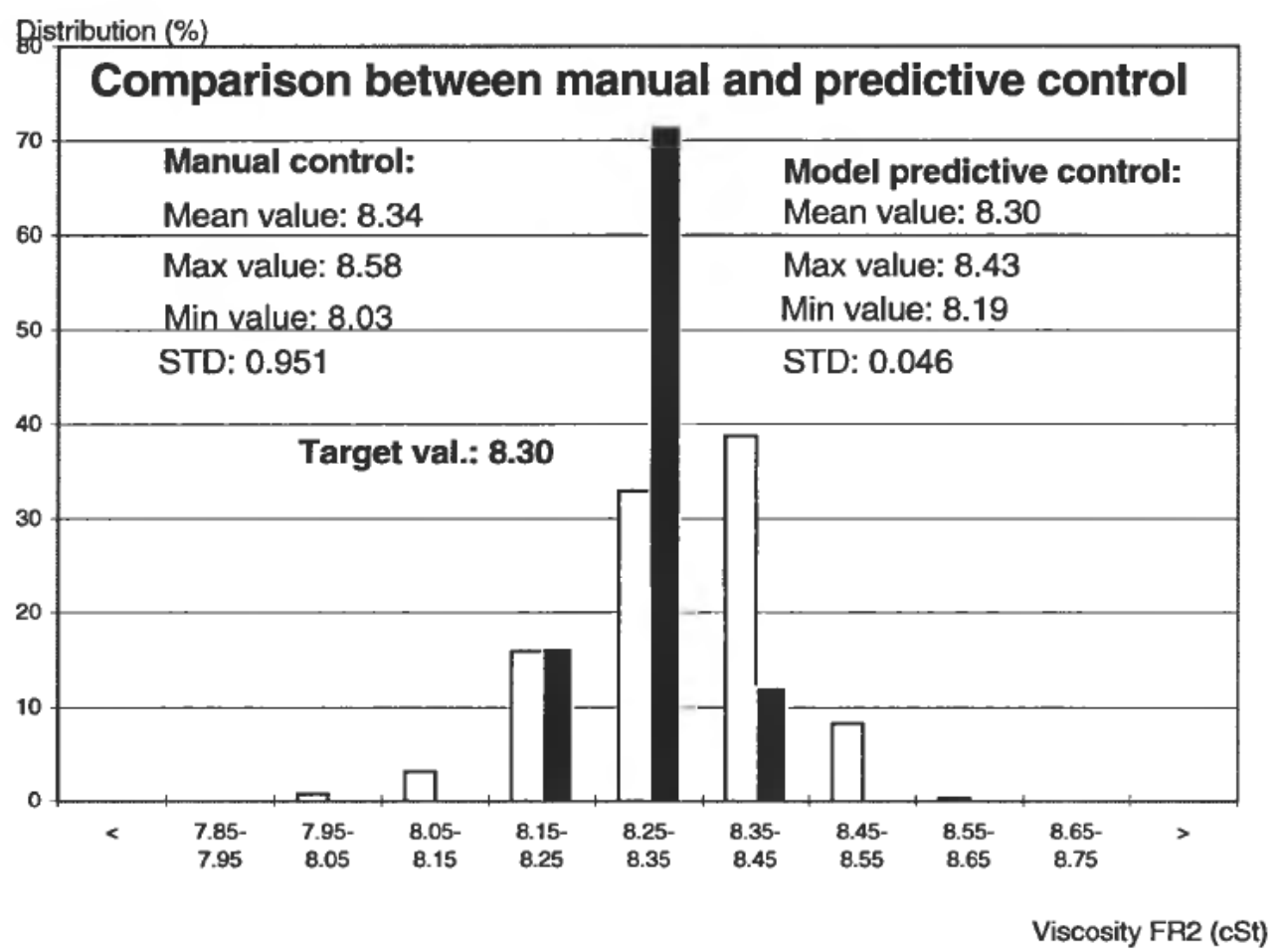

Figure 4. Comparison of manual (white columns) and model based predictive control (dark columns). 
In addition to the control described above, engineers at Nynäshamn have developed an application which maximises feed to the column, with important constraints in the condenser at the top of the column, in the two pump-arounds, and the requirement for having liquid on all trays below the feed plate. The changes in feed are implemented through D-MPC, which minimises the effect of the feed changes on the product yield and quality specifications.

The system integration between the different computer systems and programs was undertaken by the Nynäshamn-based company PRONYX.

\section{Results}

D-MPC has been in operation since the summer of 1995. The implementation went without significant problems, and the system has been well received by the operators.

Previously, the plant was operated manually, with the aid of some simple SPC-based techniques. The improvements due to model-based control are dramatic, as illustrated in Fig. 4, which compares the distribution of measured viscosity of fraction 2 with manual control to the distribution obtained with model predictive control. Standard deviation in product viscosities have been reduced by up to a factor of 25 . In addition to reducing variations in product quality, the model predictive control also provides the operators with the facility to continuously maximise throughput with minimal effect on the product qualities. Savings also result from reduced energy costs due to the improved control. The total investment cost for this project was approximately $250 \mathrm{~K}$ USD, and the annual financial benefits are approximately:

- Increased yield of most desirable fraction: $110 \mathrm{~K}$ USD.

- Increased feed rate: $120 \mathrm{~K}$ USD.

- Decreased energy consumption: 20K USD. 